\title{
Effect of Viscous Dissipation on MHD Free Convection Flow over an Inclined Plate Embedded In a Porous Medium with Heat Absorption
}

\author{
Siva Reddy Sheri ${ }^{1}$, Prasanthi Modugula ${ }^{2}$ \\ ${ }^{1}$ Department of Mathematics, GITAM University, Hyderabad Campus, Telangana, India) \\ ${ }^{2}$ Department of Mathematics, CMRTC, Telangana, India)
}

\begin{abstract}
This work is devoted to investigate the influence of viscous dissipation on the magneto hydro dynamic (MHD) free convection flow of an electrically conducting incompressible viscous fluid over an inclined plate embedded in a porous medium with heat absorption. The dimensionless governing equations for this investigation are solved numerically by using Finite element method. With the help of graphs, the effects of the various important parameters entering into the problem on the velocity, temperature and concentration are discussed. The effects of the pertinent parameters on the skin friction, Nusselt number and Sherwood number at the wall are presented numerically in tabular form. In addition, the results obtained show that these parameters have significant influence on the flow. Finally present numerical results are compared with the existing literature and there is an excellent agreement between the results.
\end{abstract}

Keywords: FEM, Heat absorption, Inclined plate, MHD, Viscous dissipation.

\section{Introduction}

The convective heat and mass transfer flows in an inclined plate embedded in a porous medium has many engineering and geophysical applications such as chemical industry, geothermal reservoirs, drying of porous solids, thermal insulation, enhanced oil recovery, MHD power generators, packed-bed catalytic reactors, cooling of nuclear reactors and underground energy transport. Chamkha [1] discussed unsteady MHD convective heat and mass transfer past a semi-infinite vertical permeable moving plate with heat absorption. Nonlinear macrokinetics of heat and mass transfer and chemical or electrochemical reactions has explored by Sieniutycz [2]. Conjugate effects of heat and mass transfer on MHD free convection flow over an inclined plate embedded in a porous medium have investigated by Farhad Ali et al. [3].

There has been a renewed interest in studying magneto hydro dynamic (MHD) flow and heat transfer in porous and non-porous media due to the effect of magnetic fields on the boundary layer flow control and on the performance of many systems using electrically conducting fluids. In addition, this type of flow has attracted the interest of many investigators in view of its applications in many engineering problems such as MHD generators, plasma studies, nuclear reactors, geothermal energy extractions. The effect of an electromagnetic field on natural convection in an inclined porous medium was reported by Bian et al. [4]. Chamkha et al. [5] studied unsteady MHD free convective heat and mass transfer from a vertical porous plate with hall current, thermal radiation and chemical reaction effects.

Natural convection heat transfer induced by internal heat generation has recently received considerable attention because of numerous applications in geophysics and energy-related engineering problems. Acharya and Goldstein [6] studied numerically two-dimensional natural convection of air in an externally heated vertical or inclined square box containing uniformly distributed internal energy sources. Their numerical results showed two distinct flow pattern systems depending on the ratio of the internal to the external Rayleigh numbers. Also, it was found that the average heat flux ratio along the cold wall increased with increasing external Rayleigh numbers and decreasing internal Rayleigh numbers. Rapits [7] examined mathematically the case of time varying two-dimensional free convective flow of an incompressible, electrically conducting fluid along an infinite vertical porous plate embedded in a porous medium. Recently, Radiation Effects on MHD free convection flow along vertical flat plate in presence of joule heating and heat generation was reported by Mohammad mokaddes ali et al. [8].

The chemical reaction effect depends on the heterogeneous or homogeneous of the reaction. And this effect depends on the occurrence at an interface a single- pharse volume reaction. A reaction is said to be of the order $\mathrm{n}$, if the reaction rate is proportional the $\mathrm{n}$-th power of the concentration. When, the rate of the reaction is directly proportional to the concentration itself, such reactions are of the first order. If we consider air or water, these don't occur naturally but mixed with foreign mass. This foreign mass is responsible for chemical 
reactions. The study of such chemical reaction is necessary for the importance of chemical technologies, such as food processing and polymer production etc. The effect of a chemical reaction on an unsteady flow past an impulsively started vertical plate which is subjected to uniform mass flux and in the presence of heat transfer investigated by Muthucumaraswamy and Ganesan [9]. Another situation which can arise in practice is a chemical reaction produced in the porous medium. The main aspects occurring in the modeling of a chemical reaction in a porous medium are discussed by Nield and Bejan [10]. Soret and Dufour effects on transient MHD flow past a semi infinite vertical porous plate with chemical reaction discussed by Anand rao and Shivaiah [11].

In most of the studies mentioned above, viscous dissipation is neglected. viscous dissipation is the irreversible process by means of which the work done by a fluid on adjacent layers due to the action of shear forces is transformed into heat i.e., viscous dissipation changes the temperature distribution by playing a role like an energy source, which leads to affect heat transfer rates. The merit of the effect of viscous dissipation depends on whether the sheet is being cooled or heated. The influence of viscous dissipation and radiation on unsteady MHD free convection flow past an infinite heated vertical plate in a porous medium with time dependent suction has investigated by Israel Cookey et al. [12]. Lie group analysis of heat and mass transfer effects on steady MHD free convection flow past an inclined surface with viscous dissipation presented by Gnaneswara Reddy [13].

In view of the above discussions, authors envisage to investigate the effect of viscous dissipation on MHD free convection flow over an inclined plate embedded in a porous medium with heat absorption. The flow governing partial differential equations are transformed into ordinary differential equations which are then solved numerically using Finite element method. The effects of various pertinent parameters are explained with the help of graphs and tabular form. Obtained results are compared with relevant results in the existing literature and are found to be in good agreement.

\section{Formulation of The Problem}

Let us consider the unsteady flow of an incompressible viscous fluid past an infinite inclined plate with variable heat and mass transfer. The $x^{\prime}$ axis is taken along the plate with the angle of inclination $\alpha$ to the vertical and the $y^{\prime}$-axis is taken normal to the plate. The viscous fluid is taken to be electrically conducting and fills the porous half space $y^{\prime}>0$. A uniform magnetic field of strength $B_{0}$ is applied in the $y^{\prime}-$ direction transversely to the plate. The applied magnetic field is assumed to be strong enough so that the induced magnetic field due to the fluid motion is weak and can be neglected. According to Cramer and Pai [14], this assumption is physically justified for partially ionized fluids and metallic liquids because of their small magnetic Reynolds number. Since there is no applied or polarization voltage imposed on the flow field, the electric field due to polarization of charges is zero. Initially, both the fluid and the plate are at rest with constant temperature and $T_{\infty}^{\prime}$ constant concentration $C_{\infty}^{\prime}$. At time $t^{\prime}=0$, the plate is given a sudden jerk, and the motion is induced in the direction of flow against the gravity with uniform velocity $u_{0}$. The temperature and concentration of the plate are raised linearly with respect to time. Since the plate is infinite, all physical variables are functions of $y^{\prime}$ and $t^{\prime}$ only. The physical model and coordinates system is shown in Figure1.

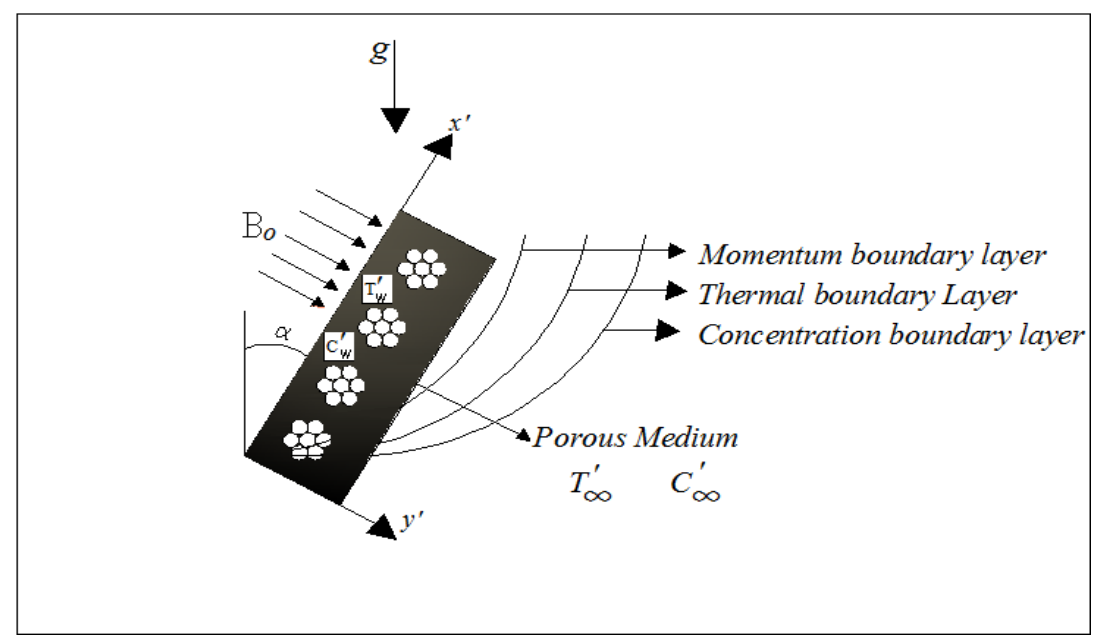

Figure- 1.The physical model and coordinates system 
Effect of viscous dissipation on MHD free convection flow over an inclined plate embedded in a ..

In view of the above assumptions, as well as of the usual Boussinesq's approximation, the governing equations reduce.

\section{Momentum equation:}

$\frac{\partial u^{\prime}\left(y^{\prime}, t^{\prime}\right)}{\partial t^{\prime}}=v \frac{\partial^{2} u^{\prime}\left(y^{\prime}, t^{\prime}\right)}{\partial^{2} y^{\prime 2}}-\left(\frac{\sigma B_{0}^{2}}{\rho}+\frac{v}{k}\right) u^{\prime}\left(y^{\prime}, t^{\prime}\right)+g \beta_{T}\left(T^{\prime}-T_{\infty}^{\prime}\right) \cos (\alpha)+g \beta_{C}\left(C^{\prime}-C_{\infty}^{\prime}\right) \cos (\alpha)$

\section{Energy equation:}

$\rho c_{p} \frac{\partial T^{\prime}}{\partial t^{\prime}}=k_{1} \frac{\partial^{2} T^{\prime}}{\partial^{2} y^{\prime 2}}-\frac{\partial q_{r}}{\partial y^{\prime}}-q_{c}\left(T^{\prime}-T_{\infty}^{\prime}\right)+\frac{v}{\rho c_{p}}\left(\frac{\partial u^{\prime}}{\partial y^{\prime}}\right)^{2}$

Species equation:

$\frac{\partial C^{\prime}}{\partial t^{\prime}}=D \frac{\partial^{2} C^{\prime}}{\partial y^{\prime 2}}-k_{2}\left(C^{\prime}-C_{\infty}^{\prime}\right)$.

where $u^{\prime}$ is the axial velocity, $T^{\prime}$ is the temperature of the fluid, $C^{\prime}$ is the species concentration, $q_{r}$ is the radiation heat flux, $x^{\prime}$ and $y^{\prime}$ are the dimensional distances along and perpendicular to the plate, $t^{\prime}$ is the time,

$\sigma$ is the electrical conductivity, $v=\frac{\mu}{\rho}$ ( $\mu$ is the viscosity and $\rho$ the constant density of the fluid) is the kinematic viscosity, $k>0$ is the permeability of the porous medium, $\mathrm{g}$ is the acceleration due to gravity, $\beta_{T}$ is the coefficient of thermal expansion, $\beta_{C}$ is the coefficient of concentration expansion, $c_{p}$ is the specific heat at constant pressure, $k_{1}$ is the thermal diffusivity, $q_{c}$ is the volumetric heat generation or absorption, $D$ is the mass diffusibility and $k_{2}$ is the chemical reaction constant.

The initial and boundary conditions are:

$u^{\prime}\left(y^{\prime}, 0\right)=0, T^{\prime}\left(y^{\prime}, 0\right)=T_{\infty}^{\prime} ; C^{\prime}\left(y^{\prime}, 0\right)=C_{\infty}^{\prime}, y^{\prime}>0$,

$u^{\prime}\left(\infty, t^{\prime}\right)=0, T^{\prime}\left(\infty, t^{\prime}\right)=T_{\infty}^{\prime} ; C^{\prime}\left(\infty, t^{\prime}\right)=C_{\infty}^{\prime}, t^{\prime}>0$.

Where $A=\frac{u_{0}^{2}}{v}$ we adopt the Roseland approximation for irradiative flux $q_{r}$ namely

$$
\begin{aligned}
& u^{\prime}\left(0, t^{\prime}\right)=u_{0}, \\
& T^{\prime}\left(0, t^{\prime}\right)=T_{\infty}^{\prime}+\left(T_{w}^{\prime}-T_{\infty}^{\prime}\right) A t^{\prime}, \\
& C^{\prime}\left(0, t^{\prime}\right)=C_{\infty}^{\prime}+\left(C_{w}^{\prime}-C_{\infty}^{\prime}\right) A t^{\prime}, t^{\prime}>0, \\
& q_{r}=-\frac{4 \sigma_{0}}{3 k_{3}} \frac{\partial T^{\prime 4}}{\partial y^{\prime}},
\end{aligned}
$$

where $\sigma_{0}$ is the Stefan-Boltzmann constant and $k_{3}$ is the mean absorption coefficient.

We assume that the temperature differences within the flow are sufficiently small and $T^{\prime 4}$ can be expressed as a linear function of the temperature. This is accomplished by expanding $T^{\prime 4}$ in a Taylor series about $T_{\infty}^{\prime}$ and neglecting the higher order terms, we get

$$
T^{\prime 4} \approx 4 T_{\infty}^{\prime 3} T^{\prime}-3 T_{\infty}^{\prime 4} \text {. }
$$

Substitution equations (5) and (6) in equation (2), yields to

$$
\rho c_{p} \frac{\partial T^{\prime}}{\partial t^{\prime}}=k_{1}\left(1+\frac{16 \sigma_{0} T_{\infty}^{\prime 3}}{3 k_{1} k_{3}}\right) \frac{\partial^{2} T^{\prime}}{\partial y^{\prime 2}}-q_{c}\left(T^{\prime}-T_{\infty}^{\prime}\right)+\frac{v}{\rho c_{p}}\left(\frac{\partial u^{\prime}}{\partial y^{\prime}}\right)^{2}
$$

Introducing the following dimensionless variables

$y=\frac{u_{0}}{v} y^{\prime}, u=\frac{u^{\prime}}{u_{0}}, t=\frac{u_{0}^{2}}{v} t^{\prime}, \theta=\frac{T^{\prime}-T_{\infty}^{\prime}}{T_{w}^{\prime}-T_{\infty}^{\prime}}, \phi=\frac{C^{\prime}-C_{\infty}^{\prime}}{C_{w}^{\prime}-C_{\infty}^{\prime}}$ 
Equations (1), (3) and (7) reduce to

$$
\begin{aligned}
& \frac{\partial u}{\partial t}=\frac{\partial^{2} u}{\partial y^{2}}-\left(M+\frac{1}{K}\right) u+G r \theta \cos (\alpha)+G m \phi \cos (\alpha) \\
& \operatorname{Pr} \frac{\partial \theta}{\partial t}=(1+N) \frac{\partial^{2} \theta}{\partial y^{2}}-Q \theta+E c\left(\frac{\partial u}{\partial y}\right)^{2} \\
& \frac{\partial \phi}{\partial t}=\frac{1}{s c} \frac{\partial^{2} \phi}{\partial y^{2}}-K r \phi
\end{aligned}
$$

Where

$$
\begin{aligned}
& M=\frac{\sigma B_{0}^{2} v}{\rho u_{0}^{2}}, \frac{1}{K}=\frac{v^{2}}{u_{0}^{2} k}, G r=\frac{g \beta_{T} v\left(T_{w}^{\prime}-T_{\infty}^{\prime}\right)}{u_{0}^{3}}, G m=\frac{g \beta_{c} v\left(C_{w}^{\prime}-C_{\infty}^{\prime}\right)}{u_{0}^{3}}, \\
& \operatorname{Pr}=\frac{\mu c_{p}}{k}, N=\frac{16 \sigma_{0} T_{\infty}^{\prime 3}}{3 k_{1} k_{3}}, Q=\frac{q_{c} v t_{0}}{k}, E c=\frac{U_{0}^{2}}{c_{p}\left(T_{w}^{\prime}-T_{\infty}^{\prime}\right)}, S c=\frac{v}{D}, K r=\frac{k_{2} v}{u_{0}^{2}}
\end{aligned}
$$

Here, $M$ is the magnetic parameter called Hartmann number, $K$ is the permeability parameter, $G r$ is the thermal Grashof number, $G m$ is the mass Grashof number, Pr is the Prandtl number, $N$ is the radiation parameter, $Q$ is heat absorption, $E c$ is Eckert number, $S c$ is the Schmidt number and $\mathrm{Kr}$ is the dimensionless chemical reaction parameter. The corresponding initial and boundary conditions (4), become

$$
\begin{aligned}
& t \leq 0: u(y, t)=0, \theta(y, t)=0, \phi(y, t)=0, y>0, \\
& t>0: u(0, t)=1, \theta(0, t)=t, \phi(0, t)=t, \\
& u(y, t) \rightarrow 0, \theta(y, t) \rightarrow 0, \phi(y, t) \rightarrow 0, y \rightarrow \infty .
\end{aligned}
$$

\section{Method of Solution}

The finite element method is the most versatile numerical procedure employed by engineers in solving linear and nonlinear partial differential equation systems. The Finite element method has been implemented to obtain numerical solutions of equations (9)-(11) under boundary conditions (13). Finite element method by Bathe[15] and Reddy [16] have been utilized in diverse branches of applied mechanics and fluid dynamics including combustion, geo mechanics, structural dynamics, magneto-hydrodynamics, bio-fluid dynamics and many other areas of technology. The basic steps involved in the finite-element analysis of a problem are as follows:

\section{Step 1: DISCRETIZATION OF THE INFINITE FLUID DOMAIN INTO FINITE ELEMENTS}

i. The whole domain is divided into a finite number of sub domains, which is called the discretization of the domain. Each sub domain is called an element. The collection of elements is then denoted the finite-element Mesh. The intersection of any two elements is termed an inter-element boundary. The intersection points are called the global nodes.

ii. The nodes and elements are numbered.

iii. The geometric properties needed for the problem are generated, which indicates the position of the elements.

\section{Step 2: DERIVATION OF ELEMENT EQUATIONS}

i. A typical element is isolated from the mesh and the variation formulation of the given problem over the typical element is constructed.

ii. An approximate solution of the variation problem is assumed. Substituting it into the element equations is made.

iii. The element matrix or stiffness matrix is constructed by using the element interpolation functions. The interpolation functions depend on the type of element (geometry, number of nodes and number of primary unknowns per node). These functions have to be derived or selected and are not already available in the literature. 
Effect of viscous dissipation on MHD free convection flow over an inclined plate embedded in a ..

\section{Step 3: ASSEMBLY OF ELEMENT EQUATIONS}

The algebraic equations so obtained are assembled by imposing the inter element continuity conditions (i.e. the values of the nodal variables at the nodes are identical for two or more elements). This yields a large number of algebraic equations known as the global finite element model, which governs the whole domain.

Step 4: IMPOSITION OF BOUNDARY CONDITIONS

The initial and final boundary conditions (12) are imposed on the assembled equations.

Step 5: SOLUTION OF ASSEMBLED EQUATIONS

The assembled equations are solved by any of the numerical technique viz. Gaussian Elimination, Gauss-Seidel, LU decomposition etc.

Now, it is important to calculate the physical quantities of primary interest, which are the Skin friction, Nusselt number and Sherwood number. The expressions for Skin-friction, Nusselt number Sherwood number are given by

Skin-friction coefficient $\tau=-\left[\frac{\partial u(y, t)}{\partial y}\right]_{y=0}$

Nusselt number $N u=\left[\frac{\partial \theta(y, t)}{\partial y}\right]_{y=0}$

Sherwood number $S h=\left[\frac{\partial \phi(y, t)}{\partial y}\right]_{y=0}$

\section{Validation of Numerical Results}

To check the accuracy of the numerical method and code used for the solution of the problem under consideration, it was validated with the Farhad Ali et al. [3] in the absence of viscous dissipation and heat absorption. This comparison shows good agreement between the results exists. This lends confidence into the numerical results to be reported subsequently.Tables (1) to (3) represent the comparison of present values of Skinfriction $\tau$, Nusselt number $N u$ and Sherwood number $S h$ with Farhad Ali et al. [3]. By clear observation Table 1 displays the effect of various parameters on Skin friction $\tau$. From this table it is observed that the Skin friction $\tau$ increases with increasing the values of $\operatorname{Pr}, M, S c, K r$ and decreasing the values of $K, G r, G m$. On the other hand Skin friction $\tau$ decreases with increasing the values of $\alpha, N, t$. Table 2 conveys the influence of Prandtl number on Nusselt number. From this table it is clear that the Nusselt number increases on increasing the Pr. Table 3 explains the effect of Schmidt number, chemical reaction parameter and time on Sherwood number. From this table it is clear that Sherwood number increases on increasing $S c, K r$ and $t$.

Table 1: Comparison of Skin friction $\tau$ (when $E c=0$ and $Q=0$ ) with Farhad Ali et al. [3]

\begin{tabular}{|c|c|c|c|c|c|c|c|c|c|c|c|}
\hline $\operatorname{Pr}$ & $M$ & $K$ & $G r$ & $G m$ & $N$ & $S c$ & $K r$ & $\alpha$ & $t$ & Farhad Ali et al.[3] & Present Results \\
\hline 0.71 & 2 & 0.1 & 6 & 6 & 3 & 0.6 & 1 & $\pi / 6$ & 1 & 3.046 & 3.046 \\
\hline 7 & 2 & 0.1 & 6 & 6 & 3 & 0.6 & 1 & $\pi / 6$ & 1 & 3.094 & 3.094 \\
\hline 0.71 & 15 & 0.1 & 6 & 6 & 3 & 0.6 & 1 & $\pi / 6$ & 1 & 4.686 & 4.686 \\
\hline 0.71 & 2 & 0.01 & 6 & 6 & 3 & 0.6 & 1 & $\pi / 6$ & 1 & 9.930 & 9.930 \\
\hline 0.71 & 2 & 0.1 & 0 & 6 & 3 & 0.6 & 1 & $\pi / 6$ & 1 & 3.271 & 3.271 \\
\hline 0.71 & 2 & 0.1 & 6 & 0 & 3 & 0.6 & 1 & $\pi / 6$ & 1 & 3.239 & 3.239 \\
\hline 0.71 & 2 & 0.1 & 6 & 6 & 15 & 0.6 & 1 & $\pi / 6$ & 1 & 3.031 & 3.031 \\
\hline 0.71 & 2 & 0.1 & 6 & 6 & 3 & 40 & 1 & $\pi / 6$ & 1 & 3.171 & 3.171 \\
\hline 0.71 & 2 & 0.1 & 6 & 6 & 3 & 0.6 & 15 & $\pi / 6$ & 1 & 3.072 & 3.072 \\
\hline 0.71 & 2 & 0.1 & 6 & 6 & 3 & 0.6 & 1 & $\pi / 3$ & 1 & 2.26 & 2.26 \\
\hline 0.71 & 2 & 0.1 & 6 & 6 & 3 & 0.6 & 1 & $\pi / 6$ & 6 & 0.674 & 0.674 \\
\hline
\end{tabular}

Table2: Comparison of Nusselt number $N u$ (when $E c=0, N=0, t=1$ and $Q=0$ )With Farhad Ali et al. [3]

\begin{tabular}{|c|c|c|}
\hline Pr & Farhad Ali et al. [3] & Present Results \\
\hline 0.7 & 0.472035 & 0.472035 \\
\hline 1 & 0.564189 & 0.564189 \\
\hline 3 & 0.977205 & 0.977205 \\
\hline 7 & 1.492705 & 1.492705 \\
\hline 10 & 1.784124 & 1.784124 \\
\hline
\end{tabular}


Effect of viscous dissipation on MHD free convection flow over an inclined plate embedded in a ..

Table 3 Comparison of Sherwood number $S h$ (when $E c=0$ and $Q=0$ ) with Farhad Ali et al. [3]

\begin{tabular}{|c|c|c|c|c|}
\hline$S c$ & $K r$ & $t$ & Farhad Ali et al. [3] & Present Results \\
\hline 2 & 1 & 0.5 & 1.30 & 1.30 \\
\hline 3 & 1 & 0.5 & 1.60 & 1.60 \\
\hline 2 & 2 & 0.5 & 1.47 & 1.47 \\
\hline 2 & 1 & 0.8 & 1.78 & 1.78 \\
\hline
\end{tabular}

\section{Results And Discussion}

In order to attain a physical insight into the problem, we have carried out numerical solution for the field of velocity, temperature, concentration by using Finite element method. Results are displayed with the help of graphical illustrations and are shown in Figures. 2-8. Computations were carried out for various values of the physical parameters. In the present investigation, we have taken the following default parameters for the numerical calculations $G r=6.0, G c=6.0, M=2.0, K=0.1, \operatorname{Pr}=0.71, N=3.0, Q=1.0$, $E c=0.01, S c=0.2$, and $K r=1.0$.

Figure .2 shows the combined effects of thermal Grashof number $G r$, mass Grashof number $G m$, magnetic parameter $M$ and porosity parameter $K$ on the velocity profiles as shown by curves I toV respectively, The comparison of Curves I and II plotted to shows the effects of Grashof number $G r$ on the velocity profiles. We have chosen the different values of Grashof number $G r=6,10$. It is clear from the comparision of curves I and II shows that thermal Grashof number $G r$ is directly praportional to the velocity due to the improvement in buoyancy forces. Thermal Grashof number $G r$ signifies the relative importance of buoyancy force to the viscous hydrodynamic force. Increase of Grashof number $G r$ indicates small viscous effects in the momentum equation and subsequently, causes increase in the velocity profiles. Also, the comparison of Curves I and III, we found that $G m$ increase along with increase of velocity similar to $G r$. The modified Grashof number $G m$ defines the ratio of the species buoyancy force to the viscous hydrodynamic force. Thus, there is increase in fluid velocity demariating a distinctive peak value as a result of increased species buoyancy force. In this process velocity distribution reaches a maximum value in the neighborhood of the plate and then decreases properly to get into the free stream value. Furthermore, curves I and IV are plotted to show the influence of Hartmann number $M$ on velocity profiles.It is observed from the comparision of curves I and IV that velocity decreases when $M$ is increased. It is acceptable because the application of transverse magnetic field always results in a resistive type force called Lorentz force which is similar to drag force and tends to resist the fluid motion, ultimately reducing its velocity. Finally, the comparison of Curves I and V plotted to show the different values of permeability parameter $K$, it is observed that velocity is directly praportional to porosity parameter $K$. We expected the similar manner, because when we increase the permeability, it increases the size of the pores inside the porous medium due to which the drag force decreases and hence velocity increases.

Figure.3. displays the effects of $\operatorname{Pr}, N, Q$ and $E c$ on velocity in the case of cooling of the plate $(G r>0)$ as shown by Curves I toV, respectively. Here the curves I and II mentioned the effects of $\operatorname{Pr}$ on velocity. From the graph it is clear that if Princreases then velocity decreases. It is due to the fact that $\operatorname{Pr}$ with high velocity will have high viscousity. Physically, it is true due to the fact that an increase in Prandtl number $\mathrm{Pr}$ increases the viscosity of the fluid, becomes thick and consequently leads to a decrease in the velocity.Curve III shows the influence of radiation parameter $N$ on the velocity profile. From the comparison of Curves I and III, we observed that the velocity increases as $N$ increases. Physically, it is true, as higher radiation occurs when temperature is higher and finally the velocity rises. The curve IV shows the effect of Heat absorption parameter $Q$ on velocity profile. By the comparision of Curves I and IV represents, if Heat absorption parameter $Q$ increases then velocity decreases. The effect of Eckert number $E c$ on velocity profile is shown by curve V. Eckert number is the ratio of the kinetic energy of the flow to the boundary layer enthalpy difference. From the comparision of curves I and V, we found that if Eckert number $E c$ increases then velocity increases.

Figure.4 is simultaneously shows the effects of $S c, K r, \alpha$ and $t$ on velocity profiles. Comparing the Curves I and II, we found that the fluid velocity decreases as $S c$ increases. The Schmidt number embodies the ratio of the momentum to the mass diffusivity. The Schmidt number therefore quantifies the relative effectiveness of momentum and mass transport by diffusion in the Hydrodynamic (velocity) and concentration (species) boundary layers. If Schmidt number increases then velocity decreases. The values of $S c=0.2,0.6$

International Conference on Recent Innovations in Civil \& Mechanical Engineering $\quad 114 \mid$ Page [i-CAM2K16] DOI: 10.9790/1684-1605302109117 
Effect of viscous dissipation on MHD free convection flow over an inclined plate embedded in a ..

are chosen for hydrogen and water vapour in air respectively, in the case of heating of plate $(G r<0)$. It is clearly observed that velocity for hydrogen is more than that for water vapour. Physically, it is justified because for large $S c$, the fluid becomes more dense.

The effects of Prandtl number $\operatorname{Pr}$, Radiation parameter $N$, Heat absorption $Q$ and Eckert number $E c$ on temperature field are shown in Figure.5. By the comparision of the curves I and II shows that the effect of Prandtl number Pron temperature profile. It is clearly from graph the prandtl number Pr increases with temperature decreases. The curve III indicates the influence of radiation parameter $N$ on the velocity profile, by the comparison of Curves I and III, we observed the Radiation parameter $N$ is directly praportional to temperature. Curve IV shows the influence of Heat absorption $Q$ on the temperature profile, by the comparison of curves I and IV, we observed that if Heat absorption parameter $Q$ increases then temperature decreases. Curve $\mathrm{V}$ mention the effect of Eckert number $E c$ on temperature profile. Eckert number is the ratio of the kinetic energy of the flow to the boundary layer enthalpy difference. It embodies the conversion of kinetic energy into internal energy by work done against the viscous fluid stresses. The positive Eckert number $E c$ implies cooling of the plate i.e., loss of heat from the plate to fluid. From the graph we observed that, the comparision of curves I and $\mathrm{V}$ shows that if Eckert number $E c$ is directly praportional to temperature.

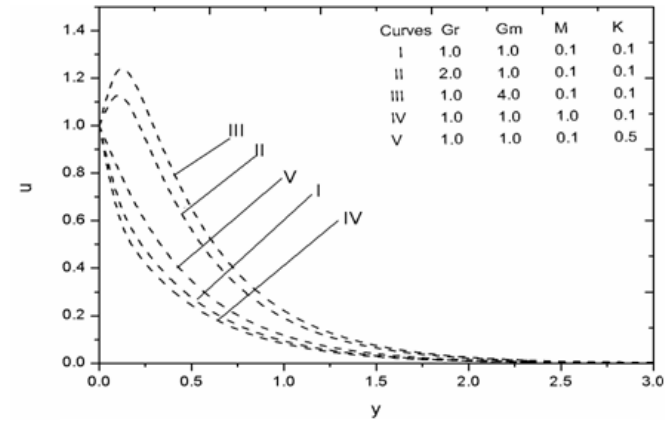

Figure- 2.Velocity profiles for different values of $G r, G m, M$ and $K$

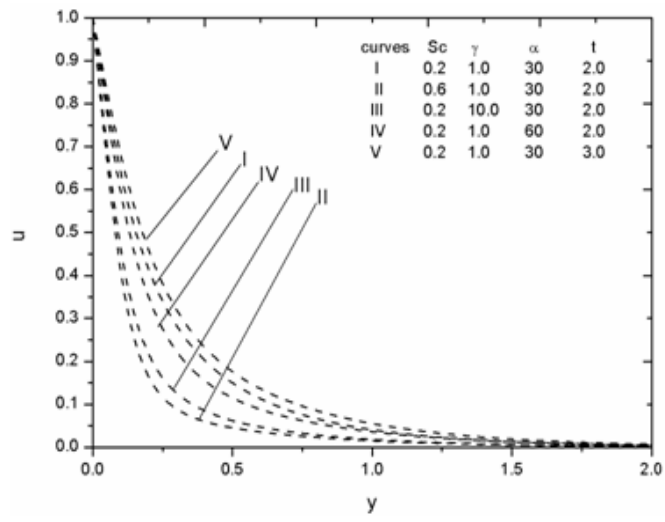

Figure- 4.Velocity profiles for different $S c, \gamma, \alpha$, and $t$

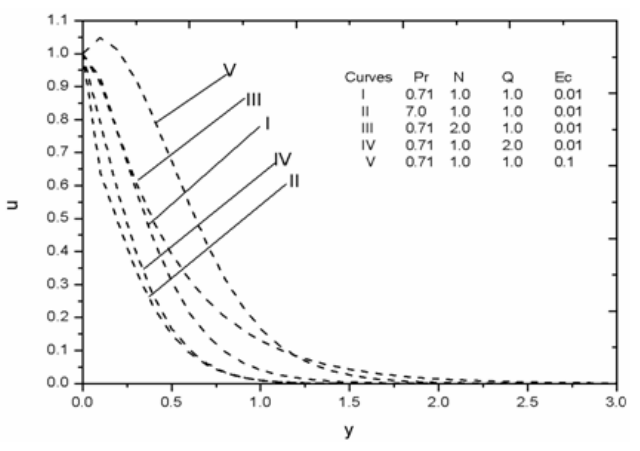

Figure-3.Velocity profiles for different values of $\operatorname{Pr}, N, Q$ and $E c$

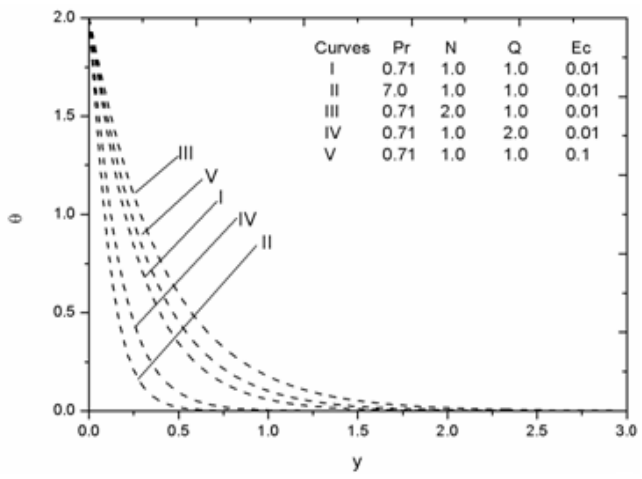

Figure- 5.Temperature profiles for $\operatorname{Pr}, N, Q, E c$ 


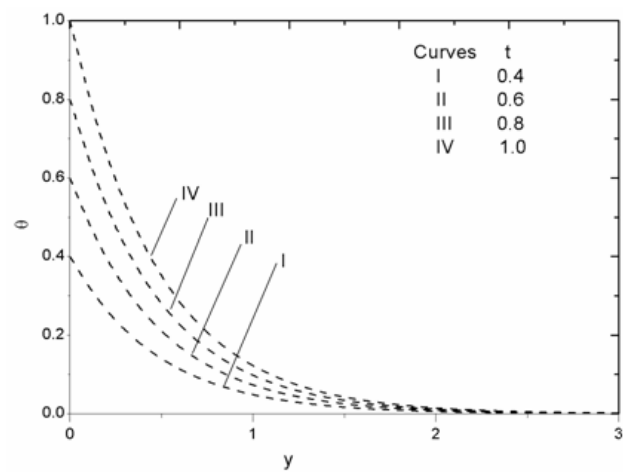

Figure-6. Temperature profiles for different values of $t$

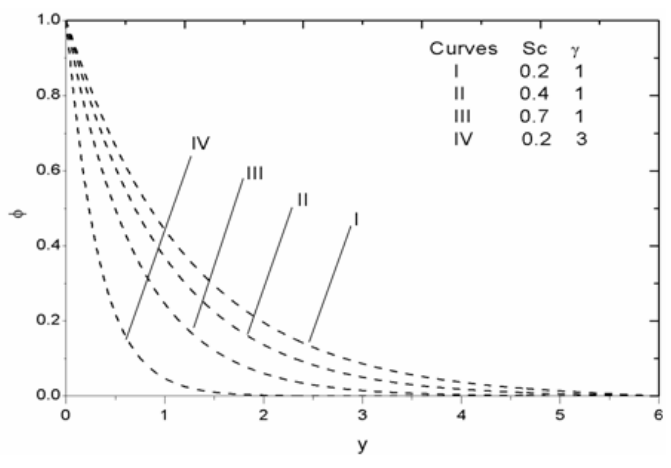

Figure-7. Concentration profiles for different values of Sc and $\gamma$

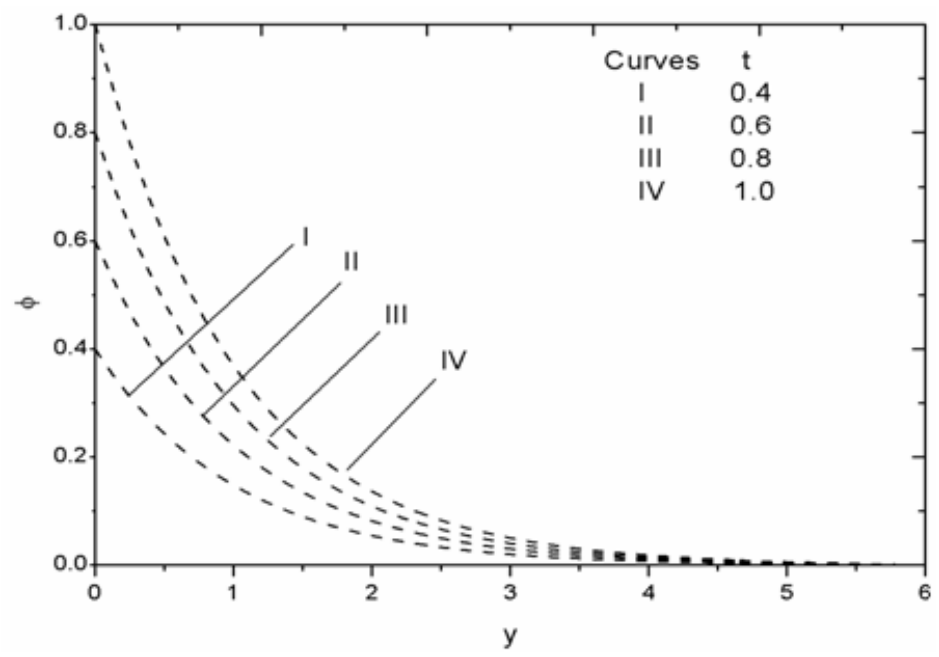

Figure- 8 .Concentration profiles for different values of $t$

Figure. 6 illustrates the effects of the dimensionless time $t$ on the temperature profile by taking four different values of time $t=0.4,0.6,0.8$ and 1.0 . From the graph it is clear that the temperature is directly praportional to time. This graphical behavior of temperature can be also checked from the boundary condition of the temperature field shown in Eq. 13. By verifying the accuracy, we conform that the analytical result for the temperature is correct.

Figure.7 displays the influence of Schmidt number $S c$ and chemical reaction parameter $K r$ on concentration profiles. By the comparison of the Curve I with Curves II \& III, it is to be noticed that if Schmidt number $S c$ increases then concentration decreases. This is due to the fact that concentration buoyancy effects to decrease yielding a reduction in the fluid velocity. The reductions in the concentration profiles are accompained by simultaneous reductions in concentration boundry layers. Furthermore, it is interesting to note that the concentration profiles fall slowly and steadily for Hydrogen $(S c=0.2)$ and Helium $(S c=0.3)$ but falls very rapidly for water vapors $(S c=0.6$ ). This is verified physically, due to the fact that the water vapors can be used for maintaining normal concentration field whereas Hydrogen can be used for maintaining effective concentration field. The physical effect of chemical reaction parameter $K r$ is observed from the comparison of Curves I \& IV, which clearly indicates that concentration profiles decrease rapidly when $K r$ is increased.

Finally, The four different values of $t=0.4,0.6,0.8$ and 1.0 of concentration profiles as shown in

Figure. 8. Infer that the concentration increases when $t$ is increased. And also, this figure provides a check of our analytical solution for the concentration field which is found to be in concord with boundary condition given in Eq. (13). 


\section{Conclusions}

In this work, we have numerically studied the effect of heat absorption and viscous dissipation on the magneto hydrodynamic (MHD) free convection flow of an electrically conducting incompressible viscous fluid over an inclined plate embedded in a porous medium. The method of solution was obtained by Finite element method. The numerical results are discussed through graphs and tabular forms for different values of material parameters entering into the problem. In addition, the results obtained showed that these parameters have significant influence on the flow, heat and mass transfer. The main findings can be summarized as

$>$ Permeability parameter $K$, thermal Grashof number $G r$, mass Grashof number $G m$, radiation parameter $N$, Eckert number $E c$ and time $t$ accelerate fluid velocity. Whereas angle of inclination $\alpha$, magnetic parameter $M$, Prandtl number $\operatorname{Pr}$, heat absorption $Q$, Schmidt number $S c$ and chemical reaction parameter $K r$ having reverse influence on velocity.

$>$ Eckert number $E c$, radiation parameter $N$ and time $t$ speed up fluid temperature. On the other hand Prandtl number $\operatorname{Pr}$, heat absorption $Q$ are having retarding effect on fluid temperature.

$>$ Concentration profile increases with increase in time $t$ whereas, concentration decreases with increase in Schmidt number $S c$ and chemical reaction parameter $K r$.

\section{References}

[1]. Chamkha AJ. Unsteady MHD convective heat and mass transfer past a semi-infinite vertical permeable moving plate with heat absorption. International Journal of Engineering Science 2004; 42: 217-230. doi:10.1016/S00207225(03)00285-4.

[2]. Sieniutycz S. Nonlinear macrokinetics of heat and mass transfer and chemical or electrochemical reactions. International Journal of Heat and Mass Transfer 2004;7(3):515-526. DOI:10.1016/S0017-9310(03)00412-5.

[3]. Farhad Ali, Ilyas Khan, Samiulhaq and Sharidan Shafie. Conjugate Effects of Heat and Mass Transfer on MHD Free Convection Flow over an Inclined Plate Embedded in a Porous Medium. Exact Analysis of Heat and Mass Transfer 2013; 8(6). doi:10.1371/journal.pone.0065223.

[4]. Bian WP, Vasseur and Meng F. Effect of an electromagnetic field on natural convection in an inclined porous medium. Int. J. Heat Fluid Flow 1996;17(1):36-44. http://dx.doi.org/10.1016/0142-727X(95)00070-7.

[5]. Chamkha AJ, Mansour MA, Abdelraheem Aly. Unsteady MHD free convective heat and mass transfer from a vertical porous plate with hall current, thermal radiation and chemical reaction effects. Int. J. Numer. Meth. Fluids 2009 ;65(4): 432-447. Doi:10.1002/fld.2190.

[6]. Acharya S, Goldstein RJ. Natural convection in an externally heated vertical or inclined square box containing internal energy sources. ASME Journal of Heat Transfer 1985;107:855-866. doi:10.1115/1.3247514

[7]. Rapits AA. Flow through porous medium in the presence of a magnetic field. International journal of energy research 1986;10(1): 97-100. Doi:10.1002/er.4440100112.

[8]. Mokaddes Ali Mohammed, Mamun AA, Md. Abdul Maleque, Nur Hosain Md Ariful Azim. Radiation Effects on MHD free convection flow along vertical flat plate in presence of joule heating and heat generation. Procedia Engineering 2013; 56: 503-509. doi:10.1016/j.2013.03.153.

[9]. Muthucumaraswamy R, Ganesan P. First order chemical reaction on flow past an impulsively started vertical plate with uniform heat and mass flux. Acta Mech 2001;147:45-57.

[10]. Nield DA, Bejan A. Convection in porous media $2^{\text {nd }}$ edition. Springer, New York.;1999.

[11]. Anand Rao J, and Sivaiah S. Soret and Dufour effects on transient MHD flow past a semi infinite vertical porous plate with chemical reaction. Journal of Naval Architecture Marine Engineering 2011;8(4):37-48.

[12]. Israel-Cookey C, Ogulu A, Omubo-Pepple VB. Influence of viscous dissipation and radiation on unsteady MHD free convective flow past an infinite heated vertical plate in porous medium with time dependent suction. Int. J. Heat Mss Transfer 1972; 46: 1253-1261.

[13]. Gnaneswara Reddy M. Lie group analysis of heat and mass transfer effects on steady MHD free convection flow past an inclined surface with viscous dissipation. Journal of applied Fluid Mechanics 2013; 6(3): 397-404.

[14]. Cramer KR, Pai SI. Magneto fluid dynamics for engineers and applied physicists. McGraw-Hill Book Co New York.1973; 204-237.

[15]. Bathe K J.Finite Element Procedures, Prentice-Hall, New Jersey. 1996.

[16]. Reddy JN. An Introduction to the Finite Element Method. McGraw-Hill Book Company: New York, $3^{\text {rd }}$ Edition; 2006 